\title{
Laborübungen zur Fabrik- und Materialflußsimulation im Lehrkomplex - Fabrikplanung/Materialflußgestaltung - (Grundkonzept)
}

\author{
Prof. Dr.-Ing. Claus-Gerold Grundig, Dipl.-Ing. (FH) Dieter Hartrampf
}

\section{Problemstellung}

Aktuelle Entwicklungen in der Industriepraxis und der Wissenschaft machen deutlich, daß die Gestaltung und Steuerung effektiver Fabrik -und Materialflußprozesse den Einsatz moderner Analysemethoden sowohl im Planungs- als auch im Realisierungsstadium erforderlich macht.

Die Anwendung der Simulationstechnik zur Analyse und zielgerichteten Synthese stochastischer, dynamischer und hochvernetzter Prozesse hat sich als ein elegantes Hilfsmittel allgemein durchgesetzt, eine steigende Tendenz auch der praxisnahen Anwendung in der Industrie ist zu verzeichnen (z. B. [1] bis [5]). Deutlich wird dabei, daß neben Anwendungen für komplexe Fragestellungen in Großbetrieben in steigendem Maße spezielle, detaillierte Untersuchungen in Klein- und Mittelbetrieben relevant werden, die durch die angebotenen, relativ nutzerfreundlichen, problemspezifischen Soft- und Hard-

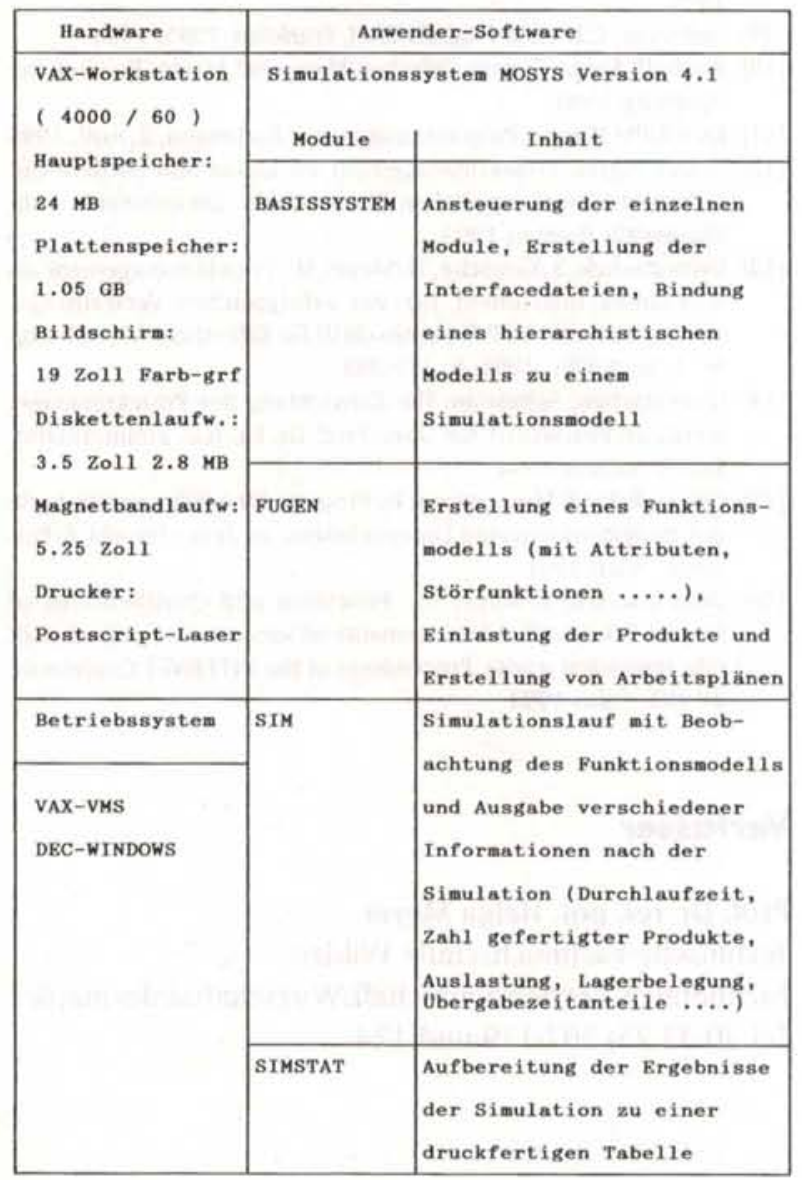

Tafel 1: Übersicht Hardware/Software des an der Technischen Fachhochschule Wildau eingeführten Simulationssystems warelösungen auch in diesen Problembereichen rationell möglich sind. Für den Lehrbetrieb in der studentischen Ausbildung im Vorlesungs- und Übungskomplex - Fabrikplanung/Materialflußgestaltung - ist es daher zwingend erforderlich, Instrumente der Simulationstechnik in ihrer Anwendung und praktischen Umsetzbarkeit den Studenten vorzustellen und anwendungsbezogen zu trainieren. Zu diesem Zweck wurde 1992 im Fachbereich Maschinenbau das Fabrikplanungssystem -MOSYS- vom Fraunhofer-Institut für Produktionsanlagen und Konstruktionstechnik (IPK) Berlin in einer speziellen Softund Hardwarekonfiguration eingeführt (Tafel 1) und im Lehrbetrieb eingesetzt.

Nachfolgend werden die Lehr- und Laborübungskonzepte sowie erste Erfahrungen vorgestellt.

\section{Lehr- und Übungskonzept}

Die Grundlagen der Simulationstechnik werden in einem speziellen Abschnitt des Vorlesungskomplexes „Fabrikplanung/Materialflußgestaltung " mit folgenden Inhaltsschwerpunkten behandelt:

- Simulationsmethoden, Grundablauf, Einsatzbereiche

- Problemstellungen bei Praxisanwendungen (Industriebeispiele)

- Anwendungsentwicklung, Simulationstechniken

- Theoretische Grundlagen, Begriffe, Prinzipien der Softwaregestaltung

- Systematik von Simulationsuntersuchungen

- Fabrik- und Materialflußprozesse als Warteschlangensysteme

- Modellbildung in der Simulationstechnik

- Entwurf von Experimentierfeldern, Ableitung von Versuchszielsetzungen

- Methoden der Verifizierung/Validierung

- Darstellung, Auswertung und Umsetzung von Simulationsergebnissen

- Grundstrukturen spezieller Simulationssysteme

- Ablauf und Umsetzung von Simulationsuntersuchungen in der Industriepraxis (Beispiele)

Der Lehransatz basiert dabei auf der Grundmethodik der Simulation, wie in Bild 1 dargestellt, und den zu trainierenden Teilkomplexen:

- Analyse Realsystem

- Modellbildung

- Simulationsexperimente

- Optimierung (Umbau) des Realsystems

Wesentlich dabei ist die Verdeutlichung der Simulationsmethode als dynamisches Analyse- und Struktu- 


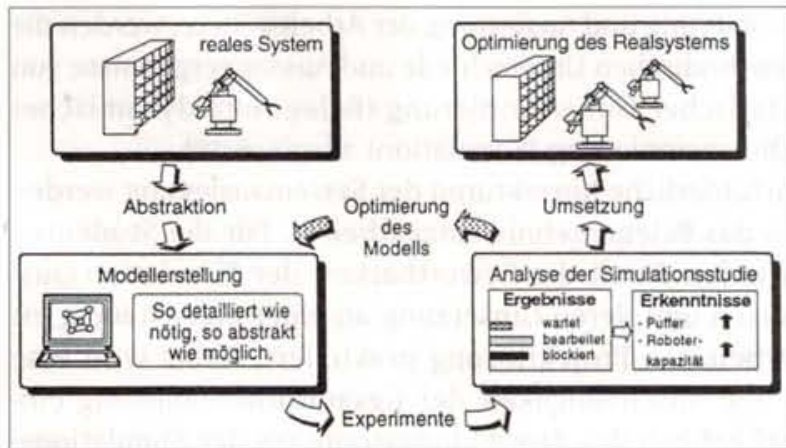

Bild 1: Grundmethodik der Simulation (nach |6|)

rierungsinstrument, daher sind diesem Vorlesungskomplex zeitlich vorgelagert die Behandlung der klassischen, konventionellen Methoden und Instrumente der statischen Fabrikplanung, zeitlich nachgelagert ist ein zusammenhängender Komplex von 5 Laborïbungen (LÜ). Diese LÜ sind inhaltlich aufeinander abgestimmt und bauen im Sinne der Erkenntniserweiterung aufeinander auf. Zur Sicherung hoher Lehr- und Übungseffekte für die Studenten werden die Laborïbungen in Gruppenstärken von 3 bis 5 Studenten mit 90 Minuten Dauer je LÜ durchgefuihrt. Basierend auf dem Vorlaufwissen der Vorlesung und den vor dem Laborübungskomplex ausgegebenen Labor- und Trainingsanleitungen durchlaufen die so vorinformierten Studenten die Übungen, wobei schrittweise der Selbsttrainingscharakter entwickelt wird.

In Tafel 2 sind die Inhaltsstrukturen der Laborübungen dargestellt. Der Konzeption dieser Übungsinhalte sind folgende Lehr- und Trainingsziele zu Grunde gelegt:

- Erkennen des Fabrik- und Werkstattsystems als offenes Warteschlangen- bzw. Bedienungssystem,

- Präzisierung von Experimentzielsetzungen,

- Verständnis zur Notwendigkeit der Abstraktion von Realprozessen und deren Darstellung im Modell,

- Modellierung von Prozessbeispielen am Modellsystem -MOSYS- (Einsatz der Funktionsbausteine, Beschreibung der Topologie, Präzisierung mit TOKEN),

- Diskussion zulässiger Abstraktionsgrade im Modell,

- Wirkungen von Störungen auf den Prozessablauf,

- Definition und Eingabe von systemgerechten Datenstrukturen (Arbeitspläne, Betriebsmittel etc.),

- Deutung und Einordnung der Simulationsergebnisse, Umsetzung auf das reale System.

- Entwurf von Experimentierstrategien, Festlegungen zu Parameter- und Untersuchungsbereichen,

- Simulation von Prozessabläufen, visuelle Erkenntnisse zu Gesetzmäßigkeiten der Warteschlangenbildung und des -abbaus, Erkenntnisse zur Vielzahl von Einflüssen und zu Wirkungszusammenhängen auf das dynamische Verhalten von Elementen sowie Ableitung und Vergleich von Kenngrößen der Prozesselemente,

- Abbildungsgenauigkeiten/Daten- und Modellüberprüfungen.

Die bisherigen Erfahrungen zeigen, daß die inhaltlichen Schwerpunkte der Laborïbungen insbesondere auf folgende Probleme anzusetzen sind:

- Abbildung realer Prozesse im Modell,

- systemgerechte Datengenerierung,

- Erkenntnisse zum Wechselverhalten von Parametern und deren Wirkùngen auf spezielle Kenngrößen,

\begin{tabular}{|c|c|}
\hline $\mathrm{LU}-\mathrm{Nr}$. & Inhal tasstruktur \\
\hline 1 & 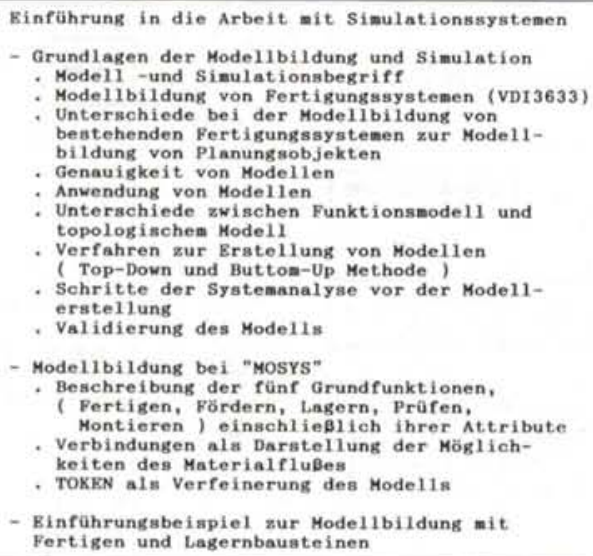 \\
\hline 2 & $\begin{array}{l}\text { Ubung zur Modellbildung an einem vorhandenen } \\
\text { System } \\
\text { - Systemanalyse des FFS-System der TFH Wildau } \\
\text { ( Drehmaschine, Bohrmaschine, Roboter, Ablage } \\
\text { für Rohteile, Ablage für Fertigteile, Leit- } \\
\text { rechner ) } \\
\text { - Uberführung der Bestandteile des Fertigungs- } \\
\text { systems in MOSYS-Funktionen } \\
\text { - Darstellung der Verbindungen (Materialflug) } \\
\text { - Bingabe des Modells in das Fabrikplanungs- } \\
\text { Bystem MoSYs, einschlieBlich der Zuweisung } \\
\text { von Attributen für die Funktionen und } \\
\text { Darstellung der Verbindungen }\end{array}$ \\
\hline 3 & 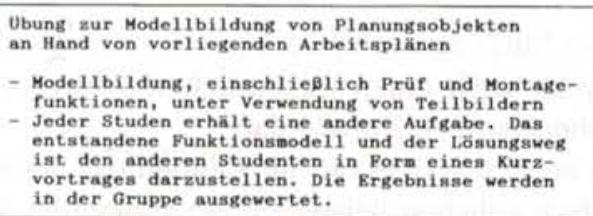 \\
\hline 4 & 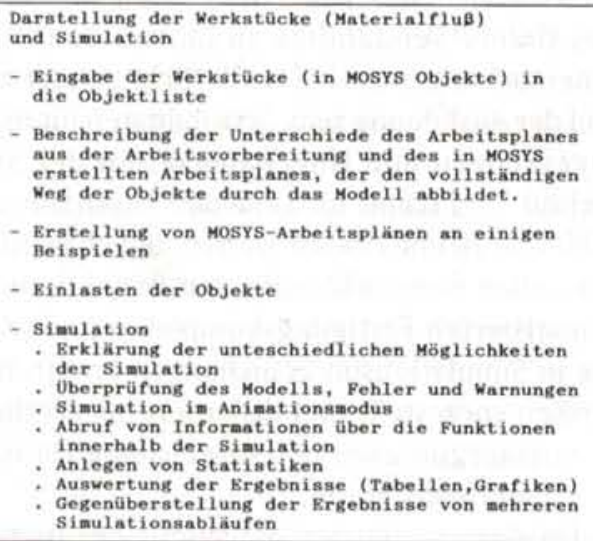 \\
\hline 5 & 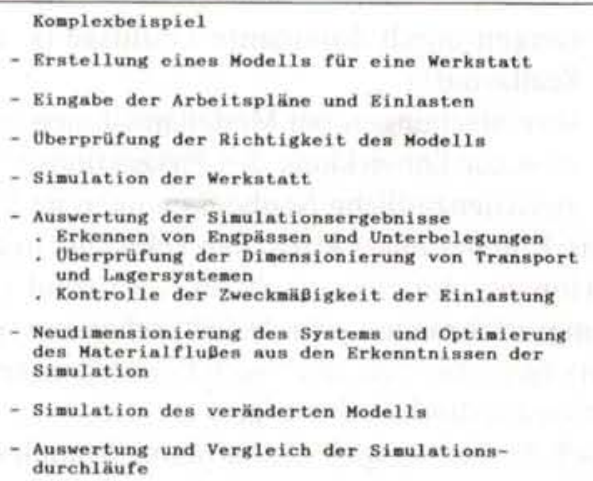 \\
\hline
\end{tabular}

Tafel 2: Inhaltsstrukturen der Laborübungen

- praxisrelevante Deutung und Umsetzung der Ergebnisse auf das Problembeispiel, praxisbezogene Formulierung von Systemeingriffen (Systemanpassungen).

In Bild 2 ist eine Realwerkstatt und in Bild 3 ihre Umsetzung in Modellstrukturen - gemäß den Modellierungsprinzipien in MOSYS [7] nach der Top-Down-Methode dargestellt. Das Training dieser Abstraktionsschritte und 


\section{EINATE}

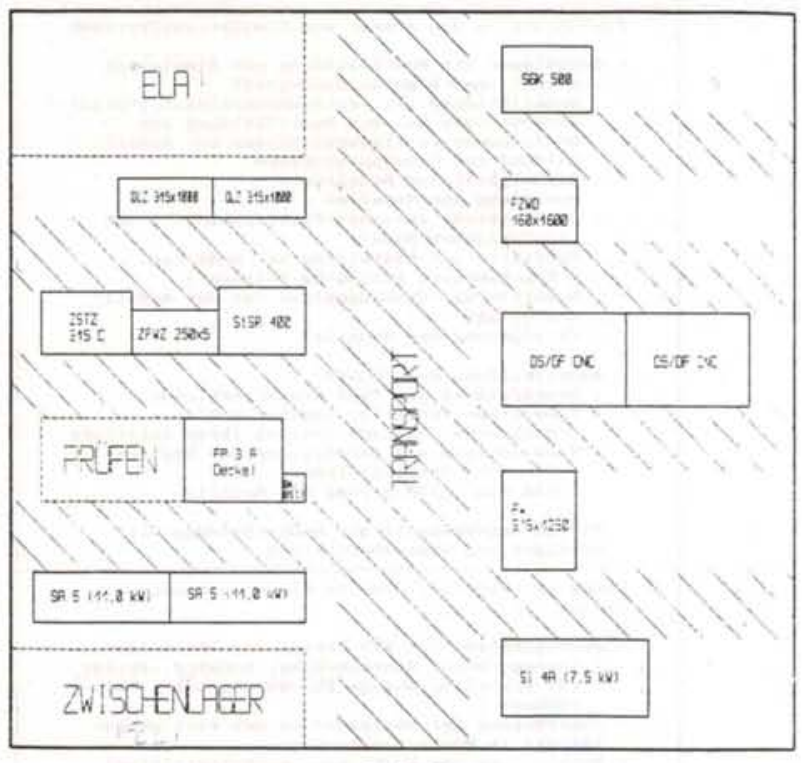

RUSEAN

Bild 2: Realstruktur einer Werkstatt

die Nutzung der systemspezifischen Abbildungsprinzipien stellt einen wesentlichen Schwerpunkt in den Laborübungen 2 und 3 dar.

Es zeigt sich, daß nur im Rahmen des Trainings an unterschiedlichen Beispielen bei steigender Komplexität das tiefere Verständnis zu (abstrakten) Simulationsuntersuchungen erzielt wird. Nicht zuletzt muß es auch Ziel der Ausbildung sein, Sensibilitäten fuir mögliche Aussagegrenzen und Ungenauigkeiten der Simulationstechnik und damit für sinnvolle Einsatzbereiche dieser Methode zu entwickeln. Diesbezüglich werden in einem speziellen Seminarkomplex zur Projektierung eines automatisierten Fertigungskomplexes (Industriebeispiel) die in Simulationsuntersuchungen ermittelten Projektgrößen vorgestellt und diskutiert hinsichtlich:

- Aussagegültigkeit und Abweichung vor und nach der Projektrealisierung,

- Überlagerungen der simulationsspezifischen Optimierungen durch dominante Einflüsse (z. B. Ideal- zu Reallayout),

- Vereinfachungen bei Modellannahmen (z. B. Annahmen zur Entwicklung des Produktionsprogrammes),

- zwischenzeitliche Neubedingungen im Realprozeß.

Das Training eines kritischen Umgangs mit den Simulationsergebnissen erscheint auf Grund von Anwendungserfahrungen erforderlich, schon um typische Einsatzmöglichkeiten, aber auch Einsatzgrenzen der Simulationsmethode aufzuzeigen.

Nach Absolvierung des Laborïbungskomplexes (LÜ 1 bis LÜ 5) werden die erzielten Erkenntnisse genutzt, um an einem im Folgesemester zu erarbeitenden Komplexbeleg „Werkstattprojektierung“ die Anwendung der Simulationsmethode an einer eigenständig projektierten Werkstattlösung zu überprïfen.

In einer Speziallaborübung (LÜ 6) wird die erarbeitete Lösung experimentell getestet. So werden z. B. Produktionsprogramme, Produktmix, Auftragsgrößen, Fördermittelanzahlen und Einschleusreihenfolgen und Auslastungen der Fördermittel gezielt variiert und analysiert. An Hand der ermittelten Kennwerte, z. B. zur Dimen- sionierung und Auslastung der Arbeitsplätze, werden die methodischen Unterschiede und Aussageergebnisse von statischer Dimensionierung (Beleg) und dynamischer Dimensionierung (Simulation) ausgewertet.

Erforderliche Korrekturen der Systemauslegung werden in das Belegergebnis eingearbeitet. Für die Studenten werden damit die Bewertbarkeit der Simulationsaussagen und deren Umsetzung an einer eigenständig erarbeiteten Projektlösung praktiziert. Dabei wird eine hohe Anschaulichkeit der Gesamtproblemlösung einschließlich des Anwendungstrainings der Simulationsmethode erzielt.

\section{Zusammenfassung}

Vorgestellt wurden das Grundkonzept einschließlich inhaltlich-methodischer Schwerpunkte der Vorlesung und des Laborübungskomplexes „Fabriksimulation“ im Lehrgebiet Fabrikplanung/Materialflußgestaltung.

Die gewonnenen Erfahrungen zeigen den hohen methodisch-didaktischen Anspruch, der dieser Lehraufgabe zukommt, zumal die theoretischen Grundlagen stark gebündelt geboten werden müssen.

Die Weiterentwicklung der Ausbildungsinhalte wird in folgenden Schwerpunkten gesehen:

- Modellbildung und Experimente bei Klein- und Großsystemen,

- Anwendung der Simulationstechnik (Diplom- und Projektarbeiten),

- Einsatz der Simulationstechnik innerhalb von PPSSystemen,

- Erarbeitung von Betriebskennlinien auf Basis von Simulationsexperimenten (Projektaufgaben).

\section{Literatur}

[1] Mertins, K.: Rabe, M.: Rechnergestiitzte Planung von Fertigungssystemen: TECHNIKA 13 (1992), S. 16-21

[2] Tagungsbericht, „Simulation und Verstehen"; Tagungsbericht 1991. ASIM - gfmt

[3] Tagungsbericht, "Simulation und Fabrikbetrieb“, Tagungsbericht 1993. ASIM - gfmt

|4| Schumann, Ch.-A.; Keil, B.: Computersimulierte Fabrik; Wissenschaftliche Zeitschrift der TU Chemnitz-Zwickau 34 (1992) 5/6, S. $31-44$

[5] Erkmann, J.; Grundig, C.-G.: „Nutzung des Projektierungssystems RPS-FAIF zur Gestaltung eines integrierten gegenstandsspezialisierten Fertigungsabschnitts"; Fertigungstechnik und Betrieb 36 (1986) 7. S. 407-411

[6] Eversheim, W.: Skudelny, Ch.; Linhoff, Ma.: „Simulation im Fabrikalltag - Einsatz, Nutzen und Perspektiven; Simulation und Fabrikbetrieb, Tagungsbericht 1993, S. 9-27

[7] MOSYS, Systemunterlagen; IPK Berlin

\section{Verfasser}

Prof. Dr-Ing. Claus-Gerold Grundig

Technische Fachhochschule Wildau

Fachbereich Maschinenbau

Telefon (0 33 75) 507-171

Dipl.-Ing. (FH) Dieter Hartrampf, Laboringenieur

Technische Fachhochschule Wildau

Fachbereich Maschinenbau

Telefon (0 33 75) 507-107, -109 oder -116 


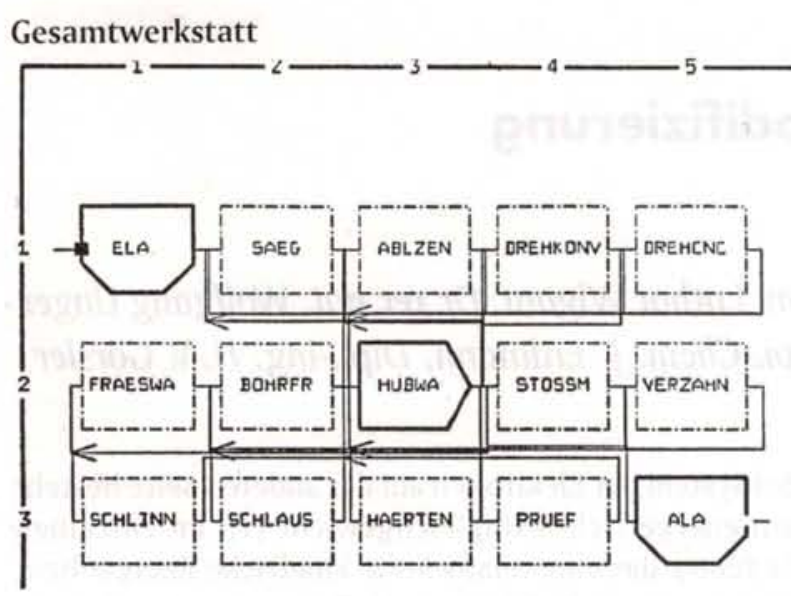

Teilprozesse

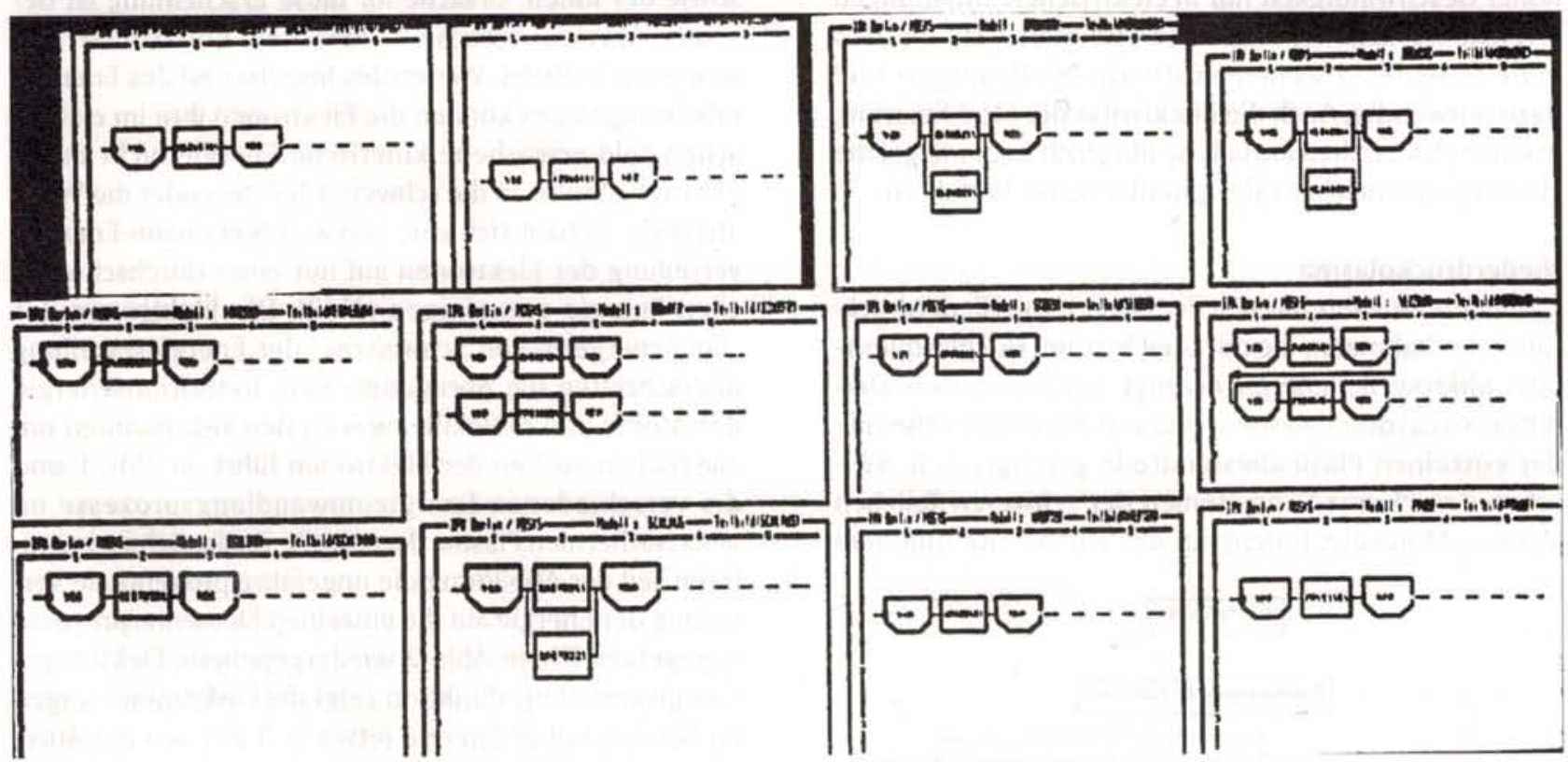

Bild 3: Ergebnis der Modellierung der Werkstatt (Komplexbild/Teilbilder) 